\title{
Comparing deep and shallow neural networks in forecasting call center arrivals
}

\author{
Andrea Manno · Fabrizio Rossi · Stefano \\ Smriglio - Luigi Cerone -
}

Received: date / Accepted: date

\begin{abstract}
Forecasting volumes of incoming calls is the first step of the workforce planning process in call centers and represents a prominent issue from both research and industry perspectives. We investigate the application of Neural Networks to predict incoming calls 24 hours ahead. In particular, a Deep Learning method known as Echo State Networks, is compared with a completely different shallow Neural Networks strategy, in which the lack of recurrent connections is compensated by a careful input selection. The comparison, carried out on three different real world datasets, reveals similar predictive performance, although the shallow approach seems to be more robust and less demanding in terms of timeto-predict.
\end{abstract}

Keywords call centers forecast - machine learning · artificial neural networks · echo state networks

\section{Introduction}

Call centers represent major components for today's organizations, with a huge market size (Statista Research Department, 2021) and millions of employees worldwide. The set of activities that a company carries out to optimize employee productivity is called Workforce management (WFM). WFM plays a key role in call center industry, as labour costs typically comprise the largest portion of the total operating budget. Furthermore, it offers a surprisingly rich application context for several operations management methodologies such as forecasting, capacity planning, queueing and personnel scheduling. In fact, there is a huge scientific literature concerning with WFM in call centers and we refer the reader to surveys by Koole and Li (2021), Aksin et al. (2007) and Gans et al. (2003). The general goal of call

\footnotetext{
A. Manno, F. Rossi, S. Smriglio

Dipartimento di Ingegneria, Scienze dell'Inofrmazione e Matematica, Università degli Studi dell'Aquila, Via Vetoio, 67100 L'Aquila, Italia Tel.:

Fax:

E-mail: andrea.manno@univaq.it, fabrizio.rossi@univaq.it, stefano.smriglio@univaq.it
} 
centers WFM is to find a satisfactory trade-off between the Level of Service (LoS) provided to customers and personnel costs.

This is a complex task due to several sources of uncertainty, such as intraday/intra-week seasonality and variability of the actual number of employees. These fluctuations cannot be managed at operational (real-time) level unless some (expensive) overcapacity has been introduced at planning level. Therefore, the first fundamental step of a successful WFM consists in making an accurate forecast of the highly uncertain incoming/outgoing call volumes.

The prediction of the incoming/outcoming call volumes in a given time horizon (for instance, one day), subdivided into equal time intervals (say one hour) can be naturally modeled as a Time Series Forecast problem (Box et al., 2015). It can refer to monthly or yearly forecasts (i.e., long-term forecasts) to guide strategic planning decisions, or to short-term forecasts, i.e., weekly, daily or hourly forecasts, mainly devoted to support staffing decisions and work-shift scheduling.

Call volume forecasts can also be categorized according to three different features: the call type (incoming or outgoing); the lead time, i.e. how much time in advance the forecast is performed with respect to the prediction time and the duration, that is, the time granularity of the forecast (e.g., hourly or daily). For example, we address the forecasting of incoming calls with 24 hours lead time and hourly duration.

Today's call centers are part of multi-channel contact centers, in which the customer, before interacting with an agent by phone, is assisted through a dedicated website, can send/receive emails and can even be engaged in a virtual conversation through an interactive chatbot. All of these interactions are a valuable source of information to support accurate call volumes forecasts and, although several researches concern time series forecasts, relatively few attempts have been made to harness these exogenous factors in call volumes prediction.

In this paper, after a review of the literature on forecasting methods in call centers ( $(2)$, we investigate two forecasting algorithms based on Neural Networks ( 33 ). These algorithms are well suited to capture the complex nonlinear relationships between the time series to be predicted and the values observed on the exogenous factors and lead to better forecasting accuracy. They embody two opposite methodological lines. The first method, proposed in Bianchi et al. (2015), is based on Echo State Networks (ESN), a Deep Learning architecture with strong representational power and very suitable for time series (see §3.1), but also highly sensitive to parameter tuning. Indeed, ESNs combine the high computational power of a deep architecture with an easy training phase, but require an expensive external optimization of the network hyperparameters.

The second method is a shallow Single Layer Feedforward Network (SLFN) (e.g., Grippo et al. (2015)), that is, a Neural Network with a simpler structure, preferable for practical implementations thanks to its stability and ease of use. In general, the structure of SLFNs is not suited to reproduce complicated temporal patterns. We show that with an appropriate input selection strategy, based on simple autocorrelation analyses ( 33.2$)$, one can make the performance of the shallow SLFN fully competitive with that of ESNs while requiring less computation workload and maintaining ease of implementation. These findings are drawn by first comparing the two appproaches on a benchmark dataset from the Machine Learn- 
ing literature $(\S 4)$, then by experimenting the methods on two publicy available call centers datasets $(\S 5)$. Finally, in $\S 6$ some concluding remarks are presented.

\section{Literature review}

Far from being exhaustive (the interested reader is referred to Gans et al. (2003); Aksin et al. (2007); Ibrahim et al. (2016)), we review some known approaches so as to highlight the main features of the various classes of forecasting models.

The process of inbound calls has been often modeled as a Poisson arrival process (see e.g., Garnett et al., 2002; Gans et al., 2003; Wallace and Whitt, 2005), by assuming a very large population of potential customers where calls are statistically independent low-probability events. However, these assumptions may not be consistent with real world call centers, characterized by evident daily and weekly seasonality. Moreover, it has been observed that call centers arrivals exibhit a relevant overdispersion (see e.g., Jongbloed and Koole, 2001; Steckley et al., 2005; Aldor-Noiman et al., 2009), in which the variance of arrivals tends to dominate its expected value.

Two typical ways to overcome the previous drawbacks are: a nonhomogeneous Poisson process, with non-constant arrival rate which is a deterministic function of the time, may be adopted to cope with the seasonalities (see e.g., Brown et al., 2005; Green et al., 2007); while a doubly stochastic Poisson process (see e.g., Avramidis et al., 2004; Liao et al., 2012; Ibrahim and L'Ecuyer, 2013), in which the arrival rate is itself a stochastic process, can be shown to have variance larger than mean (Ibrahim et al., 2016).

Although stochastic processes have the advantage of being computed analytically, they are not suited to include important relationship between the target time series and some highly correlated exogenous factors.

Moving towards time series forecasting approaches, many Authors adopted autoregressive linear models, like seasonal moving average (e.g., Tandberg et al., 1995), autoregressive integrated moving average (ARIMA) (e.g., Andrews and Cunningham, 1995; Bianchi et al., 1998; Antipov and Meade, 2002; Taylor, 2008) and Exponential Smoothing (e.g., Taylor, 2010; Barrow and Kourentzes, 2018), in order to predict future incoming calls volumes as linear combinations of past occurences of the target time series. These methods are both simple and easy to implement. Moreover they can include important external factors (like calendar effects or advertising campaigns) by considering additive/multiplicative terms, covariates and transfer functions. However, they require a certain degree of users' knowledge of the system in order to make some a priori modeling decisions. Consequently, they are not flexible with respect to environmental changes and they are not able to capture hidden and often highly nonlinear relationship involving correlated exogenous factors. For these reasons, they are more suited to forecasting regular working periods.

Only recently, to overcome the previous drawbacks, some Neural Networks (NNs) methodologies have been proposed. While poorly interpretable, NNs models possess a strong flexibility and representational power, which has stimulated the adoption of NNs prediction models in many different fields (e.g., Sun et al., 2003; Dong et al., 2018; Avenali et al., 2017). In the investigated context, NNs allow 
to automatically reproduce nonlinear interactions between the target time series and the correlated exogenous factors, exempting the user from making deliberate modeling choices. This aspect is crucial whenever exogenous factors strongly affect the target time series observations in a non-trivial way, as it is often the case in call centers arrivals. Moreover, NNs quickly adapt to changes in the system. Thus, they represent a promising instrument for inbound calls volumes forecasting in contact centers.

Millán-Ruiz et al. (2010) applied momentum backpropagation NN algorithm (LeCun et al., 2012) to forecast incoming volumes in a multi-skill call center. As NN inputs they selected some lagged observations of the incoming volumes and some static exogenous factors (like the day of the week), based on a relevance criterion computed via the Mann-Whitney-Wilcoxon test (e.g., Nachar et al., 2008). Their proposed NN approach seems to outperform the ARIMA method in all the skills' groups. Barrow and Kourentzes (2018) adopted a single layer feedforward network (SLFN) (e.g., Grippo et al., 2015) method in which sinusoidal waves and special dummy variables inputs are combined to cope with respectively the seasonalities and the outliers (like special days or singular) of the target time series (see also Kourentzes and Crone, 2010). This SLFN methodolgy showed to perform better than linear models like ARIMA and exponential smoothing, however it requires a prior knowledge of outliers periods, which are often unpredictable.

As an alternative to shallow NNs, Deep Learning (DL) methods may be considered. In particular Recurrent Neural Networks (RNNs) (e.g., Goodfellow et al., 2016) fit very well time series forecasting problems. Indeed, their feedback connections are able to reproduce temporal patterns which may repeat over time. Nonetheless, the training of RNNs is a very complicated task, due essentially to vanishing/exploding gradient phenomena as highlighted in Pascanu et al. (2013), and to bifurcation problems (see e.g., Doya, 1992).

Jalal et al. (2016) adopted an ensamble NN architecture by combining an Elman NN (ENN) (Elman, 1990), a semi-recurrent NN structured as a SLFN enriched with feedback connections where the latter may not be trained, and a Nonlinear Autoregressive Exogenous Model (NARX) NN, i.e. a SLFN in which the inputs are lagged values of the target time series and exogenous factors (Chen et al., 1990). They favorably compared their ensamble method with a time-lagged feed-forward network (a SLFN where the only inputs are lagged values of the target time series) to forecast the incoming volumes on 15 minutes intervals for an Iranian call center. However, the combination of ENN with NARX-NN does not look straightforward and also efficiency may become an issue as ENNs are known to suffer from slow training convergence (see e.g., Cheng et al., 2008).

Bianchi et al. (2015) used Echo State Networks (ESNs) to the 24 hours ahead forecast of the hourly incoming call volumes in an antenna of a mobile network, where data are taken from the Data for Development (D4D) challenge (Blondel et al., 2012). As will be better specified in Section 3, ESNs are DL methods which try to retain the benefits of RNNs while mitigating their training issues. In Bianchi et al. (2015) ESNs outperform ARIMA and exponential smoothing on the D4D dataset by automatically leveraging 6 exogenous time series (as well as the target time series), and an external genetic algorithm used to determine the better network configuration. 
Motivated by the aforementioned advantages of NNs-based methods, mainly in terms of prediction accuracy and flexibility to changes, aim of this work is to identify suitable NN-s able to leverage exogenous factors to forecast call volumes in practical settings. The first method considered is the ESN-based method by Bianchi et al. (2015). It has the high computational power of a deep architecture along with an easy training phase. However, it requires a careful and costly (in terms of CPU time) external optimization of network hyperparameters, preventing straightforward implementation by unskilled users. The second is a new NARXlike shallow SLFN approach in which a simpler and easy-to-use model is adopted. Despite its simplicity implies less representational power, we are able to combine the SLFN model with a simple but effective input selection strategy that keeps the SLFN performance competitive with respect to ESNs, speeding-up significantly the computation time and avoiding implementation awkwardness.

\section{Methodologies}

In this section we give a summary of ESNs general principles and we describe the shallow SLFN methodology proposed for the experimental comparisons, with particular focus on the lagged inputs selection.

Firstly, we introduce the considered supervised learning framework. In particular, we suppose to have a dataset of $p+1$ time series $\left(x_{1}[t], x_{2}[t], \ldots, x_{p}[t], \hat{y}[t]\right)$, with $t=1, \ldots, H . \hat{y}[t]$ is the target time series one, i.e. the time series we are aimed to predict, while $x_{1}[t], x_{2}[t], \ldots, x_{p}[t]$ are exogenous time series that may help to build the prediction model. Among the exogenous time series, some of them may have an aleatory nature (e.g. the number of outgoing calls from a call center division) and some other may be deterministic (e.g. the time series representing the timestamp in which the time series values are observed). The goal is to train a ML model to predict a lagged value of the target time series at timestamp $t+k$ with $k \in \mathbb{N}_{+}$by leveraging all observations of exogenous and target time series collected until the current timestamp $t$. Formally, let us consider a training set of $T R$ of available data

$$
T R:=\left\{\left(x_{1}[t], x_{2}[t], \ldots, x_{p}[t], \hat{y}[t], \hat{y}[t+k]\right), t=1, \ldots, H_{T R}\right\},
$$

where $H_{T R}$ is the length of the training horizon. The predictive model is trained on the basis of $T R$ and it is tested on a similar set of data, denoted as testing set $(T S)$, for which the lagged target time series values are also known but whose samples have not been used during the training phase. Generally, as it is the case here, in time series forecasting applications, the samples of $T R$ are temporarily consecutive and provided in an increasing order, and $T S$ consists in a subsets of data corresponding to the tail of the whole period of data collection, i.e.

$$
T S:=\left\{\left(x_{1}[t], x_{2}[t], \ldots, x_{p}[t], \hat{y}[t], \hat{y}[t+k]\right), t=H_{T R}+1, \ldots, H_{T R}+H_{T S}\right\},
$$

where $H_{T S}$ is the length of the testing horizon. As will be clarified later, ESNs, being dynamical systems, require that data are provided with such a temporal order both in $T R$ and $T S$, while for SLFNs this requirement is not strictly necessary for $T R$, but it is for $T S$, as a sequential rolling horizon strategy is adopted in the testing phase. 
In this work we consider hourly timestamps and a prediction advance of 24 hours, i.e. $k=24$.

\subsection{Brief summary on ESNs}

ESNs are composed of three main layers:

- a standard input layer as in SLFNs,

- a deep hidden layer, denoted as reservoir,

- a standard linear output layer, denoted as readout.

The reservoir is a chaotic layer made up of a large variety of connection patterns (including feedforward, feedback and loop connections) connecting $r$ hidden units and reproducing multiple temporal dynamics. ESNs act as a dynamic system in which, at current time step $t$, the input signal $x[t] \in \mathbb{R}^{p}$ is propagated from the input layer to the reservoir by means of feedforward weighted connections, with weights $W_{p}^{r} \in \mathbb{R}^{p \times r}$. In the reservoir, the signals pass through a sparse subset of the multiple weighted connection patterns (with weights $W_{r}^{r} \in \mathbb{R}^{r \times r}$ ), as the reservoir connectivity should be very low to avoid signal explosions after a certain number of time steps. Finally, the signals exiting the reservoir reach the output layer through further feedforward weighted connections (with weigths $w_{r}^{o} \in \mathbb{R}^{r}$ ), producing the output of the network $y[t]$. More formally, the current output $y[t]$ of a ESN is computed as follows. At first, the state of the units of the reservoir, say $h[t] \in \mathbb{R}^{r}$, is updated as

$$
h[t]=f\left(W_{p}^{r T} x[t]+W_{r}^{r} h[t-1]\right),
$$

where $W_{p}^{r T} x[t]$ is the result of propagating the current input signal $x[t]$ through the input-reservoir weighted connections, $W_{r}^{r} h[t-1]$ represents the propagation of the previous reservoir states i.e. the memory of the network, and $f: \mathbb{R}^{r} \rightarrow \mathbb{R}^{r}$ is a nonlinear function (typically sigmoidal). The values of $W_{p}^{r}$ and $W_{r}^{r}$ are randomly assigned in a previous initialization phase. Then, the current output is computed as

$$
y[t]=w_{r}^{o T} h[t] .
$$

The weights $w_{r}^{o}$ are the only trained ones. Figure 3.1 represents a schematic structure of an ESN, where the dotted connections correspond to the weights which are assigned at random in the initial phase, and the solid connection represents the only involved in the training phase. Some ESNs may have also further random feedback connections from the output layer to the reservoir, and trainable feedforward connections from the input to the output layers, but for the sake of simplicity they are not considered here.

Concerning the whole training process, consider a training set of $m$ inputoutput pairs

$$
(x[1], \hat{y}[1+h]),(x[2], \hat{y}[2+h]), \ldots,(x[m], \hat{y}[m+h]),
$$

where the input signals $x[i]$ with $i=1,2, \ldots, m$ are associated to $m$ consecutive time instants, $h$ is the a priori selected forecast lead time, and $\hat{y}[i+h]$ is the observed output at time instant $i+h$. As anticipated before, in the first initialization 


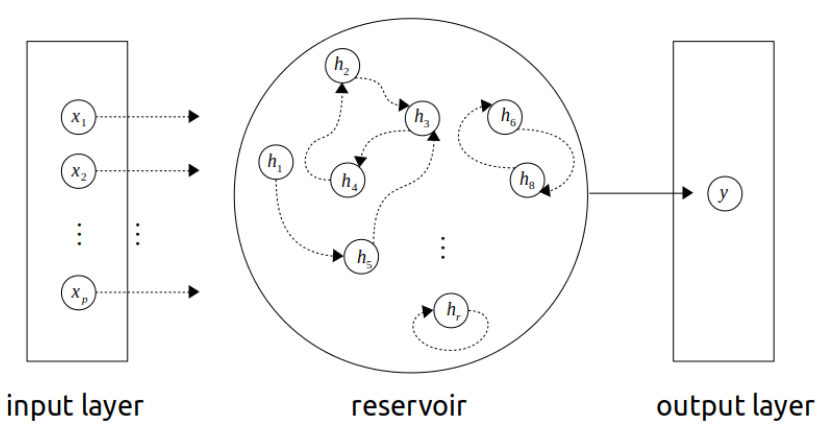

Fig. 1 A schematic depiction of a simplified ESN.

step the input and reservoir weigths $W_{p}^{r}$ and $W_{r}^{r}$ are assigned randomly (remaining unaltered during the training process), so that, in the second step denoted as warming, the sequence of reservoir states $h[1], h[2], \ldots, h[m]$ can be computed according to (3) and (4). The sequence of states can be organized into a $m \times r$ matrix $H$ as follows

$$
H=\left[\begin{array}{c}
h[1]^{T} \\
h[2]^{T} \\
\vdots \\
h[m]^{T}
\end{array}\right],
$$

and the observed outputs can be arranged into the $m$-dimensional vector $\hat{y}$ as

$$
\hat{y}=\left[\begin{array}{c}
\hat{y}[1+h] \\
\hat{y}[2+h] \\
\vdots \\
\hat{y}[m+h]
\end{array}\right] .
$$

Then, in the final learning step, the training problem can be conveniently formulated as determining the output weights $w_{r}^{o}$ as the solution of the following regularized linear least squares (RLLS) convex problem

$$
w_{r}^{o}=\underset{w \in \mathbb{R}^{r}}{\operatorname{argmin}} \frac{1}{2}\|H w-\hat{y}\|^{2}+\frac{\lambda}{2}\|w\|^{2},
$$

with $\lambda \in \mathbb{R}^{+}$.

Therefore, the ESN training involves the computationally irrelevant initialization and warming steps, and the relatively easy solution of a convex unconstrained optimization problem in the learning step. Training the readout weights can be interpreted as determining the proper linear combination of the nonlinear reservoir states in order to reproduce the desired temporal patterns of the target time series.

Alternative strategies have been considered to train the readout weights in the learning phase, for example by replacing (8) with an Elastic Net Penalty problem (Zou and Hastie, 2005) to obtain sparse solution, or to use a further ML algorithm 
like Support Vector Machines (e.g., Shi and Han, 2007). However, only the RLLS formulation is considered in this work.

Once trained, the ESN can be used to the step by step forecast associated to the future time instants.

ESNs, retaining the advantages of complex nonlinear RNNs models with a much simpler training, are well suited for the short-term forecast of time series (for long-term forecast simpler models may perform better), mainly whenever some correlated exogenous time series are provided to the model.

On the contrary, ESNs need a careful selection of many hyperparameters to produce accurate forecast. Specific attention should be devoted to the matrix of reservoir weights $W_{r}^{r}$. Although randomly determined, $W_{r}^{r}$ should respect the echo state property (Lukoševičius and Jaeger, 2009), i.e. the influence of current input signal $x[t]$ and state $h[t]$ on future states should vanish in a finite number of time steps. This property is ensured if the spectral radius of $W_{r}^{r}$, that is $\rho\left(W_{r}^{r}\right)$, satisfies

$$
\rho\left(W_{r}^{r}\right)<1 .
$$

From a practical point of view (9) can be enforced by simply rescaling $W_{r}^{r}$ after the initialization, nonetheless $\rho\left(W_{r}^{r}\right)$ should not be too far from 1 to avoid the opposite behavior, i.d. a too fast vanishing influence of the current state. It can be shown that if echo state property is satisfied, ESNs have a universal approximation property in the sense that they can realize every nonlinear filter with bounded memory arbitrarily well (Maass et al., 2007).

Further important hyperparameters are the number of the reservoir units, the reservoir connectivity (i.e. the number of nonzero weights connections) and the subset of exogenous time series to be considered as input.

To determine the optimal value of the previous hyperparameters, being crucial for the ESN performance, Bianchi et al. (2015) applied an external genetic algorithm. Such algorithmic choice is driven by the fact that the evaluation of the performance for a given combination of hyperparameters is a result of an ESN training process, so it has to be trated as a black-box function. The same genetic algorithm approach is adopted in this work.

\subsection{Shallow SLFNs with lagged inputs}

As far as for the shallow SLFN approach, a NARX strategy has been adopted in which the network is provided with lagged inputs of the target and exogenous time series, as well as with the current values of exogenous factors.

A critical point concerns the selection of the exogenous factors and of the lagged inputs to be included to obtain good quality prediction models. Differently from other sophisticated and/or time consuming approaches (e.g., Weng and Liu, 2006; Kourentzes and Crone, 2010; Karasu et al., 2020), the adopted methodology is based on automated simple filters applied to correlations and autocorrelations coefficients (Box et al., 2015), whose thresholds can be easily adapted to the specific application domain, without particular optimization/forecasting skills. The method is based on two main steps:

1. A two-phases preprocessing for exogenous factors and lagged input selection;

2. A SLFN rolling horizon training. 


\subsubsection{Preprocessing}

The preprocessing step is divided into two sequential phases denoted respectively SelectExogenous and SelectLag (see Algorithm 1).

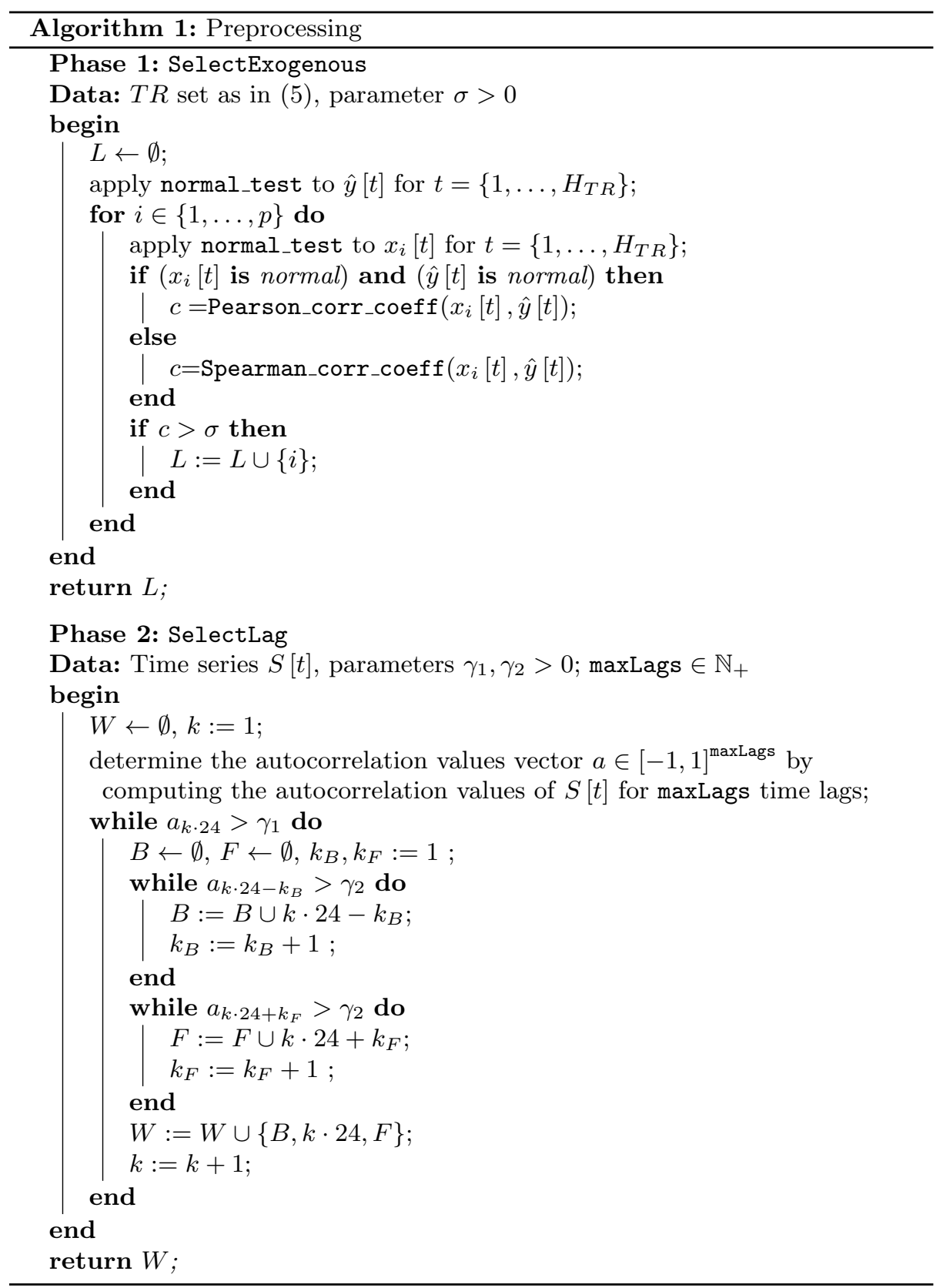

In SelectExogenous the exogenous time series are selected. In particular, a normal test is firstly applied to the target time series $\hat{y}[t]$. Then, for each exoge- 
nous time series $x_{i}[t]$ the pair $x_{i}[t], \hat{y}[t]$ is analysed and if both the series passed the normality test, their correlation coefficient $c$ is computed via the parametric Pearson test (Pearson, 1895), otherwise via the rank-order Spearman test (Zar, 2005). If $c$ is greater than a certain threshold $\sigma>0$ then $x_{i}[t]$ is selected by adding its index $i$ to the (initially empty) output list $L$.

It is worth noticing that, even if SelectExogenous can be applied to any time series, here we are interested in call centers for which time series are often not normally distributed.
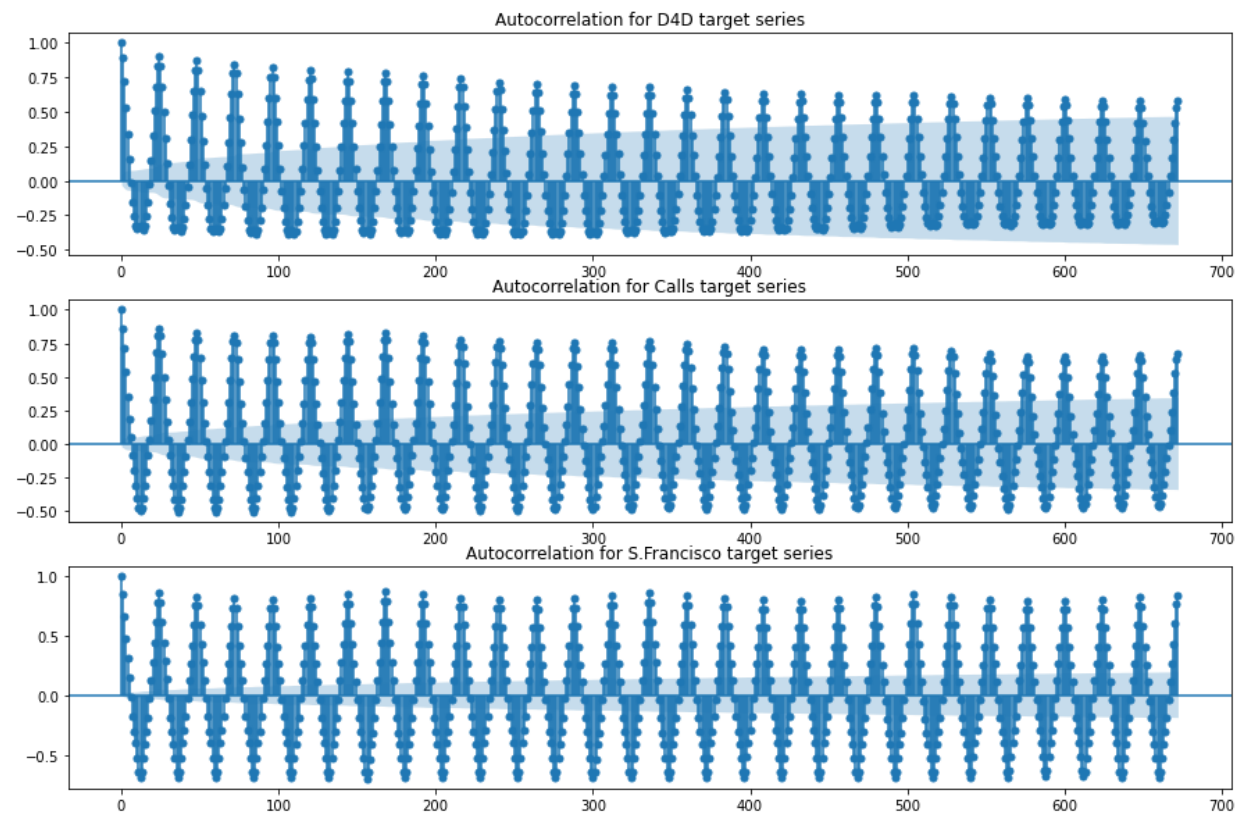

Fig. 2 The autocorrelation plots for the target time series of the investigated case studies. In the $\mathrm{x}$-axis are reported the lagged timestamps in terms of hours, while the $\mathrm{y}$-axis represent the autocorrelation value.

After SelectExogenous, SelectLag is applied to the the target time series and to the exogenous time series selected in SelectExogenous to select their lagged values to feed the SLFN. By observing the autocorrelation plot (Box et al., 2015) of this kind of time series (see e.g., Figure 2) it is possible to appreciate two different kind of intuitive temporal patterns: a daily pattern, i.e. high correlations at lags which are multiple of 24 hours, and high correlations with adjacent/proximal lags. This suggest a "time-window logic" in the selection of the lagged inputs. In particular, considering a time series, the idea is to iteratively select the past lagged values of multiple of 24 hours $(t-24, t-48, t-72, \ldots)$, referred to as daily-lags, as long as autocorrelation values are above a certain threshold $\gamma_{1}>0$. Then, for each selected daily-lag, a window is constructed by iteratively select forward and backward adjacent lags ( $F$ and $B$ in the scheme) as long as their autocorrelation values are above threshold $\gamma_{2}>0$. At the end of SelectLag a window of consecutive lagged inputs is determined for each selected daily-lag ( $W$ in the scheme). 
Notice that, for notation simplicity, the SelectLag scheme is reported for a single generic time series $s[t]$. SelectLag is not applied to the deterministic exogenous series for which lagged inputs would provide no information.

\subsubsection{SLFN rolling horizon training}

Once SelectExogenous and SelectLag have selected the exogenous factors, and the lagged values for both the target and exogenous time series, sets $T R$ and $T S$ can be built accordingly. Clearly, besides the lagged values, also the current values of the target and of the exogenous factors are included in each sample. Then, the hyperparameters of the SLFN are heuristically estimated by means of a simple grid-search method with $k$-fold cross-validation (see e.g., Bishop, 2006). Similarly to ESNs, an expensive external optimization procedure could be applied to determine the optimal values of those hyperparametrs. Nevertheless, as shown in the performed experiments, the SLFNs are more robust with respect to hyperparmaters selection, so that they obtain good performance even if a cheaper grid-search procedure is applied.

The training phase has been organized according to a rolling horizon way. In particular, at each rolling horizon step $k$ corrisponding to a certain current day $d$, a SLFN model is trained on the basis of the current training set, say $T R^{k}$ and it is used to generate the 24 predictions for day $d+1$. Then, At step $k+1$, the 24 samples associated to day $d+1$ are included in the training set, while samples form the less recent day are removed. Formally, being $A_{d}$ the set of 24 hourly samples associated to day $d, T R^{k+1}=\left(T R^{k} \backslash A_{d-H_{T R}+1}\right) \cup A_{d+1}$, and so on. In the experimental setting presented in the sequel, starting from the basic ordered and consecutive $T R$ and $T S$ in the first rolling horizon step the SLFN is trained on a training set $T R^{0}=T R$ and the predictions are generated for the 24 hours associated to the first 24 samples of $T S$, i.e. $A_{H_{T R}+1}$ (see (2)). Then, $T R^{1}=\left(T R^{0} \backslash A_{d-H_{T R}+1}\right) \cup A_{H_{T R}+1}$, the SLFN is trained again, and the predictions are produced for the hours associated to samples $A_{H_{T R}+2}$, and so on.

It is worth noticing that in the considered setting the grid-search precedure is performed only once on the initial $T R$, even if alternative choices can be considered.

\section{Benchmarking on a challenge dataset}

The two considered methodologies are first calibrated and assessed on a benchmarking dataset known as D4D.

\subsection{D4D dataset description}

$\mathrm{D} 4 \mathrm{D}$ is an open data challenge on anonymous call patterns of Oranges mobile phone users in Ivory Coast. It contains anonymized Call Detail Records (CDR) of phone calls and SMS exchanges between customers in a cell covered by an antenna of a telecommunication company. Data are collected in the period ranging from December 2011 to April 2012. The dataset is taken from Blondel et al. (2012) and, in particular, it is the one labeled with as "antenna-to-antenna traffic on an 
hourly basis". We restricted our attention on the CDR related to the antenna labeled 1. Although this dataset did not originate from a call center, its features are fully comparable with the features associated to call volumes, making the experiment meaningful and, at the same time, directly comparable with results in the literature. The time series of the $\mathrm{D} 4 \mathrm{D}$ dataset are reported in Table 1.

\begin{tabular}{l|l} 
name & description \\
\hline $\mathbf{t s 1}$ & Number of hourly incoming calls \\
$\mathbf{t s 2}$ & Volume in minutes of hourly incoming calls received by the selected antenna \\
$\mathbf{t s 3}$ & Number of hourly outgoing calls sent by the selected antenna \\
$\mathbf{t s 4}$ & Volume in minutes of hourly outgoing calls sent by the selected antenna \\
$\mathbf{t s 5}$ & Hour of the day to which this CDR refers \\
$\mathbf{t s 6}$ & Day of the week to which this CDR refers \\
\hline
\end{tabular}

Table 1 Fields description for the D4D dataset.

ts1 is the target time series, ts2, ts3 and ts4 are the exogenous aleatory time series, while ts5 and ts6 are the exogenous deterministic time series.

The dataset is organised so that data of the first 109 days are inserted in $T R$ (2616 hourly samples), while data of the last 20 days (480 hourly samples) are inserted in $T S$.

\subsection{D4D experimental settings and preprocessing outputs}

Concerning the ESN, following the standard practice adopted in Bianchi et al. (2015), a constant fictitious time series ts0 with all values set to 1 is added, acting as a bias for the individual neurons of the network. The ESN hyperparameters have been determined by the same genetic algorithm used in Bianchi et al. (2015), i.e. the one presented in Deep et al. (2009) which is able to handle both continuous and discrete variables, and whose implementation is available at https://github.com/deap. The hyperparameters optimization has been carried out by extracting a validation set consisting in the last $10 \%$ of samples of $T R$ to compute the fitness function, and training the ESN on the remaining $T R$ data. Table 2 reports the adopted values for the genetic algorithm parameters, together with their short descriptions. Table 3 reports the ESN hyperparameters optimized

\begin{tabular}{l|ll} 
param. name & description & value \\
\hline population_size & size of the population & 50 \\
n_generations & number of generation & 20 \\
n_offsprings & number of children to produce at each generation & 35 \\
mutpb & probability that an offspring is produced by mutation & 0.2 \\
cxpb & probability that an offspring is produced by crossover & 0.5 \\
mu_1 & mean of the Gaussian distribution used to mutate individuals & 0.0 \\
mu_2 & number of individuals to select for the next generation & 50 \\
parallel & enable multi-threading & false \\
\hline
\end{tabular}

Table 2 Parameters used for the genetic algorithm. 
through the genetic procedure, together with their lower and upper bounds and best value found. For details of all the hyperparameters meaning the reader is referred to Løkse et al. (2017) or to the website https://github.com/siloekse/ PythonESN where the Python ESN implementation adopted in this work is freely available. A ridge regression method has been used to train the output weights.

\begin{tabular}{l|lll} 
hyperparameter name & lower bound & upper bound & best \\
\hline connectivity & 0.25 & 0.25 & 0.25 \\
n_drop & 50 & 50 & 50 \\
input_shift & 0 & 0 & 0 \\
teacher_shift & 0 & 0 & 0 \\
n_internal_units & 100 & 500 & 429 \\
teacher_scaling & 0.1 & 0.9 & 0.758 \\
noise_level & 0.0 & 0.1 & 0.0065 \\
input_scaling & 0.1 & 0.9 & 0.349 \\
spectral_radius & 0.5 & 1.4 & 0.799 \\
feedback_scaling & 0.0 & 0.6 & 0.580 \\
regularization & 0.001 & 1.0 & 0.238 \\
\hline
\end{tabular}

Table 3 Hyperparameters spaces for ESN model.

Concerning the SLFN, it has been implemented by exploiting the MLPRegressor () method of the scikit-learn Python package. The hyperparameters of the SLFN have been obtained by a simple grid-search procedure with a 5 -fold cross-validation in which the validation set has been determined by extracting randomly the $10 \%$ of samples of $T R$. the correlation threshold $\sigma$ in SelectExogenous, has been set to 0.3 as, commonly, correlations coefficients below this value are considered not relevant (see e.g., Asuero et al., 2006). As far as for SelectLag, high correlation thresholds above the values 0.7 have been considered, i.e $\gamma_{1}=0.8$ and $\gamma_{2}=0.7$. The remarkably high $\gamma_{1}$ value is to avoid to go back too many days in the lagged input selection, which may cause potential training and applicability issues, as the autocorrelation daily pattern remains stably high for a long time (see Figure 2).

\begin{tabular}{l|lll} 
hyperparam. name & description & grid values & best \\
\hline \multirow{2}{*}{ hidden_layer_sizes } & number of hidden layer neurons & $\{25,50,100\}$ & 25 \\
alpha & regularization coefficient & $\{0,0.1,0.01,0.001\}$ & 0.1 \\
solver & loss function optimizer & $\{$ lbfgs,adam $\}$ & lbfgs \\
activation function & hidden layer neurons activation function & $\{$ logistic,tanh,relu $\}$ & tanh \\
max_iter & maximum number of optimizer iterations & $\{200,400\}$ & 200 \\
\hline
\end{tabular}

Table 4 The considered hyperparameters for the SLFN grid-search.

When applied to the $\mathrm{D} 4 \mathrm{D}$, the preprocessing phases selected all exogenous time series except for ts6, and selected lagged inputs made up of 3 values (the central, one backward and one forward) up to 9 days before.

Concerning the data normalization, all data have been normalized in the interval $[0,1]$, except for the ts5 time series which, in SLFNs, has been transformed with a one-hot-encoding. 
For ESNs, all experiments have been carried out with and without seasonal differencing all aleatory time series, as a very light first order trend can be noticed from the autocorrelation plots.

\subsection{Performance measures}

Analogously to the code used in Bianchi et al. (2015), the Normalized Root Mean Squared Error (NRMSE) has been considered as performance measure. This can be computed as

$$
\operatorname{NRMSE}(\hat{y}, y)=\frac{\sqrt{\frac{1}{n} \sum_{i=0}^{n}\left(\hat{y}_{i}-y_{i}\right)^{2}}}{\operatorname{std}(\hat{y})},
$$

where $\hat{y}$ is the vector of observed values, $y$ is the vector of predicted ones, $\operatorname{std}(\hat{y})$ is the standard deviation of $\hat{y}$, and $n$ is the number of samples of $\hat{y}$ and $y$.

However, NRMSE is not suited for operational purposes, so we have also considered a specific version of the more "interpretable" Mean Absolute Percentage Error (MAPE), denoted as daily Mean Absolute Percentage Error (dMAPE) and computed as

$$
\operatorname{dMAPE}(\hat{y}, y)=\frac{1}{n} \sum_{i=0}^{n}\left|\frac{\hat{y}_{i}-y_{i}}{M\left(d_{i}\right)}\right|,
$$

where $d_{i}$ is the day corresponding to observation $i$, and $M\left(d_{i}\right)$ is the mean of the observed values in the 24 hours associated to $d_{i}$ and computed as

$$
M\left(d_{i}\right)=\frac{1}{24} \sum_{j \in d_{i}} \hat{y}_{j} .
$$

dMAPE overcomes the "infinite error" issue affecting the MAPE. Indeed, being the latter computed as $\frac{1}{n} \sum_{i=0}^{n}\left|\frac{\hat{y}_{i}-y_{i}}{\hat{y}_{i}}\right|$, it generates huge or infinite errors when one or more observations tend to the zero value, and this may be the case in hourly call center data. Moreover dMAPE seems to be more suited for daily scheduling decisions, as normalizing the error with respect to the daily mean of the hourly calls seems to have a more relevant impact from an operational point of view.

We finally look at a third performance measure, namely, the Mean of Maximum Daily Error (MMDE), chosen for its operational relevance. It is computed as

$$
\operatorname{MMDE}(\hat{y}, y)=\frac{1}{m} \sum_{j=0}^{m} \max \_ \text {__err }_{j}(\hat{y}, y),
$$

where $m$ is the number of days associated to vectors $\hat{y}$ and $y$, and max_p_err ${ }_{j}(\hat{y}, y)$ is the maximum daily percentage error associated to day $j$, say $d^{j}$, and computed as

$$
\max \_ \text {p_err }_{j}=\max \left\{\left|\frac{\hat{y}_{i}-y_{i}}{M\left(d_{i}\right)}\right|: i \in d^{j}\right\} \text {. }
$$

Being an upper bound on the forecasting error, MMDE is a good measure for the reliability of the prediction models. 


\subsection{Numerical results on D4D}

The results of the experiments on the D4D dataset are reported in Table 5. Since both the ESNs and SLFNs trained models vary, in general, with the initialization, all the reported results are averaged on 30 runs characterized by different random selections of the reservoir weights for ESNs, and different random starting solutions for the SLFNs training optimization problem.

\begin{tabular}{r|rrr} 
D4D & NRMSE & dMAPE \% & MMDE \% \\
\hline & & & \\
ESN & 0.3769 & 17.60 & 54.27 \\
SLFN & $\mathbf{0 . 3 6 9 1}$ & $\mathbf{1 6 . 7 5}$ & $\mathbf{5 0 . 6 5}$ \\
SN & 0.3909 & 17.82 & 60.89 \\
\hline
\end{tabular}

Table 5 Comparison results for D4D.

The performance of the ESN and SLFN models are compared with those of a simple Seasonal Naive (SN) (see e.g., Barrow and Kourentzes, 2018) with seasonal cycle equal to 24 , in which the 24 hourly predictions of a certain day correspond to the 24 values observed in the previous day. The motivation of comparing with SN is to verify if the more complicated NNs-based models are able to leverage exogenous factors to improve the predictive performance with respect to an extremely simple prediction model requiring no computation. Moreover, as also specified below, all target time series considered here exhibit a low correlation with the day of the week and a high correlation with the hour of the day, and this may imply good performance of the SN model with a 24 hours seasonal cycle.

All the experiments have been carried out on a laptop Intel(R) Core(TM) i7-6700HQ CPU @ 2.60G with 8 GB of RAM.

The reported results for the ESN correspond to the seasonal differencing case as they were slightly better than the ones without differencing.

First of all we notice that the NRMSE obtained with ESN are comparable with those reported in Bianchi et al. (2015) on the same dataset. Concerning the three performance, SLFN achieves always the best ones and SN the worst ones. However, a little surprisingly, the performance difference between the more sophisticated ESN and SLFN models against the SN is not so remarkable in terms of dMAPE (around 1\%), and this can be connected with the aforementioned fact the day of the week (ts5) has no relevant correlation with the target time series, making all days quite similar. Nonetheless, a certain difference may be noticed in terms of NRMSE and mainly in terms of MMDE. Considering the latter, which is very critical from an operational point of view, ESN improves the performance up to more than $6 \%$ with respect to the SN, while SLFN up to more than $10 \%$. In terms of CPU-time, the ESN requires about 40 minutes to determine the hyperparameters through the genetic algorithm, and the SLFN grid-search about 15 minutes. For both methods, the training times are irrelevant with respect to the hyperparameter selection phase. Clearly, the SN CPU-time can be considered null. 


\section{Comparisons on call center datasets}

In this section we compare the performance of the considered forecasting techniques on two real-world datasets, which are freely available on the Kaggle website (https://www.kaggle.com/).

\subsection{Call center datasets description}

The first dataset contains incoming calls information for a tech support call center. The CDR are in the form of a dump from an Automatic Calls Distribution (ACD) software. Hence, this dataset is shortly referred to as ACD. The available data are relative to 2019 year, starting from the 1st of January to the 31th of December.

The time series of the ACD dataset are reported in Table $6 . \mathbf{t s} 1$ is the target time series, time series from ts 2 to ts5 are the aleatory exogenous ones, while ts 6 and ts7 the deterministic.

By considering that a subset of data related to the final weeks of the year have been removed as their values were anomalous (probably due to the holidays), the final training set $T R$ corresponds to 258 days (6192 hourly samples) and the testing set $T S$ corresponds to 45 consecutive days (1080 hourly samples).

The second dataset, which is referred to a SF, contains information about incoming calls to a San Francisco police switchboard. The SF dataset spans across different years, starting from March 2016 to July 2019. The dataset has been selected to see how the models behave with a bigger data set. The time series involved in SF are reported in Table 7. ts1 is the target time series, time series from ts2 to ts 4 are the aleatory exogenous ones, while ts5 and ts6 the deterministic.

The final training set $T R$ corresponds to 1095 days (26280 hourly samples) and the testing set $T S$ corresponds to 193 consecutive days (4632 hourly samples).

\begin{tabular}{r|r} 
time series name & description \\
\hline ts2 & Number of hourly handled calls by all the operators in the call center. \\
ts3 & Number of hourly answered calls by all the operators in the call center. \\
ts4 & Number of hourly routed or queued per operator. \\
ts5 & Number of hourly received calls by satisfying. \\
ts6 & Hour of the day to which this CDR refers. \\
ts7 & Day of the week to which this CDR refers.
\end{tabular}

Table 6 Fields description for the ACD dataset. 


\begin{tabular}{r|r}
\hline time series name & description \\
\hline ts2 & Number of hourly received calls belonging to category Passing Call. \\
ts3 & Number of hourly received calls belonging to category Traffic Stop. \\
ts4 & Number of hourly received calls belonging to other categories. \\
ts5 & Hour of the day to which this CDR refers. \\
ts6 & Day of the week to which this CDR refers. \\
\hline
\end{tabular}

Table 7 Fields description for the SF dataset.

5.2 Call centers experimental settings and preprocessing outputs

The experimental settings are the same as those described in Section 4 .

SelectExogenous discarded the time series related to the day of the week for both ACD and SF, while SelectLag selected lagged inputs made up of windows of 3 values and up to 9 days before for ACD and 44 for SF.

The hyperparameters determined (by the genetic algorithm) for the ESN are reported in Table 8, while the ones obtained by the grid-search for the SLFN are reported in Table 9 .

\begin{tabular}{l|ll} 
hyperparameter name & ACD & SF \\
\hline connectivity & 0.25 & 0.25 \\
n_drop & 50 & 50 \\
input_shift & 0 & 0 \\
teacher_shift & 0 & 0 \\
n_internal_units & 375 & 436 \\
teacher_scaling & 0.493 & 0.211 \\
noise_level & 0.0009 & 0.003 \\
input_scaling & 0.886 & 0.196 \\
spectral_radius & 0.557 & 1.000 \\
feedback_scaling & 0.137 & 0.282 \\
regularization & 0.115 & 0.372 \\
\hline
\end{tabular}

Table 8 The selected hyperparameters for the ESN model.

\begin{tabular}{l|ll} 
hyperparameter name & ACD & SF \\
\hline hidden_layer_sizes & 50 & 100 \\
alpha & 0.1 & 0.001 \\
solver & adam & adam \\
activation function & relu & relu \\
max_iter & 400 & 400 \\
\hline
\end{tabular}

Table 9 The selected hyperparameters for the SLFN model. 
5.3 Numerical results on the call center datasets

The results for the call center datasets are reported in Table 10. The best performance of the ESN (the ones reported) are obtained with the seasonal differencing for ACD and without seasonal differencing for SF.

\begin{tabular}{r|rrr} 
& NRMSE & dMAPE \% & MMDE \% \\
ACD & & & \\
ESN & 0.3834 & 21.49 & 83.23 \\
SLFN & $\mathbf{0 . 3 5 0 2}$ & $\mathbf{1 9 . 2 8}$ & $\mathbf{7 3 . 6 3}$ \\
SN & 0.4237 & 23.45 & 89.24 \\
\hline & & & \\
SF & & & \\
\hline & & 12.60 & 39.19 \\
ESN & 0.3775 & $\mathbf{1 1 . 9 7}$ & $\mathbf{3 8 . 0 4}$ \\
SLFN & $\mathbf{0 . 3 5 8 2}$ & 16.00 & 51.58 \\
SN & 0.4808 &
\end{tabular}

Table 10 Comparison results for ACD and SF datasets.

The results show that, similarly to the D4D benchmark case, the SLFN model performs better with respect to all the performance measures. In terms of dMAPE ESN and SLFN are comparable (mainly in SF) and they both perform better than SN (for both datasets SLFN improves the SN results by almost $4 \%$ ). However, the more significant difference between the NNs-based approaches and the $\mathrm{SN}$ is found in MMDE, where SLFN improves the SN performance by more than the $15 \%$ for ACD and more than $13 \%$ in SF (the impovements for the ESN are respectively of the $6 \%$ and of the $12 \%$ ). As mentioned before, a MMDE improvement is very relevant from an operational point of view, as it may incide on the personnel sizing policy. Also the NRMSE of the ESN and SLFN models are significantly better than $\mathrm{SN}$, and comparable to each other, even if SLFN is always preferable.

As reported in Table 11, the time needed to determine the models hyperparameters (which is a good approximation of the overall time-to-predict), is much less for the SLFN than for the ESN.

Summing up, by considering both the accuracy performances and the time needed to run the method, the SLFN strategy seems the most promising one.

\begin{tabular}{r|rr} 
dataset & ESN time (min.) & SLFN time (min.) \\
\hline Calls & 90 & 30 \\
\hline S.Francisco & 400 & 84
\end{tabular}

Table 11 The time needed by each method to determine the values of the hyperparameters.

Concerning the SLFN, notice that the same preprocessing parameter settings adopted for the D4D dataset, and determined by simple statistical considerations, have been used in the call centers case studies and led to promising results. This witnesses the robustness and stability of the method. 
5.4 Numerical results for outlier days

A further analysis has been performed to compare the SLFN model (which is the most performing one) with the SN model (the cheapest one) on potential outlier days for the SF dataset. Since no information about outliers days were avialble for the investigated datasets, we have tried to impute them by using simple statistics techinques. In particular a sigma-clipping procedure (e.g., Akhlaghi and Ichikawa, 2015) has been used to determine the days of $T S$ whose overall daily calls belong to the tails of an estimated normality distribution with a $95 \%$ of confidence. The normality assumption for the overall daily calls has been confimerd by a standard normality test.

The size of the investigated testing sets made this type of analysis significant only for the SF dataset, for this reason it has been applied only to the latter.

The method detected 5 outlier days out of 193, of which 4 corresponding to very low values and 1 corresponding to a very high value. The results reported in Table 12, suggest that the performance difference between the SLFN model and the SN one tends to increase for difficult-to-handle outliers days.

\begin{tabular}{r|rr} 
SF outliers & dMAPE \% & MMDE \% \\
\hline SLFN & $\mathbf{1 3 . 0 2}$ & $\mathbf{3 6 . 8 0}$ \\
SN & 19.24 & 54.87 \\
\hline
\end{tabular}

Table 12 Comparison on the outliers days for SF.

\section{Concluding remarks}

Exploiting exogenous factors may be an option to improve the accuracy of call forecasts in call centers, mostly in modern multi-channel contact centers where many such factors are available. We have shown that both ESNs and SLFNs are able to leverage exogenous time series to improve the 24 hours ahead forecast accuracy of hourly incoming calls with respect to the simple SN method, which only uses data of the target time series. The improvement is particularly significant for the Mean of Maximum Daily Error (MMDE), which is a relevant indicator for staffing decisions. Moreover, the performance gap between the SLFN method and the SN seems to grow in the prediction of statistically detected anomalous days.

We have also documented that, implementing a careful, yet relatively simple, input selection procedure, SLFNs slightly outperform ESNs, which, in principle, have stronger computational power and are more structured to reproduce complex temporal patterns.

This outcome is also relevant in practice, as the SLFN method looks like a good candidate for industrial implementations. In fact, it is less sensitive to hyperparameter configuration, requires limited computational effort, and can properly be managed by ML non-experts. 


\section{Declarations}

Funding: no external funding.

Conflict of interest/Competing interests: no conflict of interest or competing interests.

Availability of data and material: all datasets used in the study are freely available on the websites specified in the paper.

Code availability: custom code.

Authors' contributions: Andrea Manno, Fabrizio Rossi and Stefano Smriglio contributed to the study conception and design. Andrea Manno and Luigi Cerone contibuted to data collection and code development.

\section{References}

Akhlaghi M, Ichikawa T (2015) Noise-based detection and segmentation of nebulous objects. Astrophys J Suppl S 220(1):1

Aksin Z, Armony M, Mehrotra V (2007) The modern call center: A multidisciplinary perspective on operations management research. Prod Oper Manag 16(6):665-688

Aldor-Noiman S, Feigin PD, Mandelbaum A, et al. (2009) Workload forecasting for a call center: Methodology and a case study. Ann Appl Stat 3(4):1403-1447

Andrews BH, Cunningham SM (1995) Ll bean improves call-center forecasting. Interfaces 25(6):1-13

Antipov A, Meade N (2002) Forecasting call frequency at a financial services call centre. J Oper Res Soc 53(9):953-960

Asuero AG, Sayago A, Gonzalez A (2006) The correlation coefficient: An overview. Crit Rev Anal Chem 36(1):41-59

Avenali A, Catalano G, D'Alfonso T, Matteucci G, Manno A (2017) Key-cost drivers selection in local public bus transport services through machine learning. WIT Trans Built Env 176:155-166

Avramidis AN, Deslauriers A, L'Ecuyer P (2004) Modeling daily arrivals to a telephone call center. Manage Sci 50(7):896-908

Barrow D, Kourentzes N (2018) The impact of special days in call arrivals forecasting: A neural network approach to modelling special days. Eur J Oper Res 264(3):967-977

Bianchi FM, Scardapane S, Uncini A, Rizzi A, Sadeghian A (2015) Prediction of telephone calls load using echo state network with exogenous variables. Neural Networks 71:204-213

Bianchi L, Jarrett J, Hanumara RC (1998) Improving forecasting for telemarketing centers by arima modeling with intervention. Int J Forecasting 14(4):497-504

Bishop CM (2006) Pattern recognition and machine learning. Springer

Blondel VD, Esch M, Chan C, Clérot F, Deville P, Huens E, Morlot F, Smoreda Z, Ziemlicki C (2012) Data for development: the d4d challenge on mobile phone data. arXiv preprint arXiv: 12100137

Box GE, Jenkins GM, Reinsel GC, Ljung GM (2015) Time series analysis: forecasting and control. John Wiley \& Sons 
Brown L, Gans N, Mandelbaum A, Sakov A, Shen H, Zeltyn S, Zhao L (2005) Statistical analysis of a telephone call center: A queueing-science perspective. J Am Stat Assoc 100(469):36-50

Chen S, Billings S, Grant P (1990) Non-linear system identification using neural networks. Int J Control 51(6):1191-1214

Cheng YC, Qi WM, Zhao J (2008) A new elman neural network and its dynamic properties. In: CONF CYBERN INTELL S, IEEE, pp 971-975

Deep K, Singh KP, Kansal ML, Mohan C (2009) A real coded genetic algorithm for solving integer and mixed integer optimization problems. Appl Math Comput 212(2):505-518

Dong S, Zhang Y, He Z, Deng N, Yu X, Yao S (2018) Investigation of support vector machine and back propagation artificial neural network for performance prediction of the organic rankine cycle system. Energy 144:851-864

Doya K (1992) Bifurcations in the learning of recurrent neural networks 3. learning (RTRL) 3:17

Elman JL (1990) Finding structure in time. Cognitive Sci 14(2):179-211

Gans N, Koole G, Mandelbaum A (2003) Telephone call centers: Tutorial, review, and research prospects. Manuf Serv Op 5(2):79-141

Garnett O, Mandelbaum A, Reiman M (2002) Designing a call center with impatient customers. Manuf Serv Op 4(3):208-227

Goodfellow I, Bengio Y, Courville A, Bengio Y (2016) Deep learning, vol 1. MIT press Cambridge

Green LV, Kolesar PJ, Whitt W (2007) Coping with time-varying demand when setting staffing requirements for a service system. Prod Oper Manag 16(1):13-39

Grippo L, Manno A, Sciandrone M (2015) Decomposition techniques for multilayer perceptron training. IEEE T Neur Net Lear 27(11):2146-2159

Ibrahim R, L'Ecuyer P (2013) Forecasting call center arrivals: Fixed-effects, mixedeffects, and bivariate models. Manuf Serv Op 15(1):72-85

Ibrahim R, Ye H, LEcuyer P, Shen H (2016) Modeling and forecasting call center arrivals: A literature survey and a case study. Int J Forecasting 32(3):865-874

Jalal ME, Hosseini M, Karlsson S (2016) Forecasting incoming call volumes in call centers with recurrent neural networks. J Bus Res 69(11):4811-4814

Jongbloed G, Koole G (2001) Managing uncertainty in call centres using poisson mixtures. Appl Stoch Model Bus 17(4):307-318

Karasu S, Altan A, Bekiros S, Ahmad W (2020) A new forecasting model with wrapper-based feature selection approach using multi-objective optimization technique for chaotic crude oil time series. Energy 212:118750

Koole G, Li S (2021) A practice-oriented overview of call center workforce planning. arXiv preprint arXiv:210110122

Kourentzes N, Crone SF (2010) Frequency independent automatic input variable selection for neural networks for forecasting. In: The 2010 international joint conference on neural networks (IJCNN), IEEE, pp 1-8

LeCun YA, Bottou L, Orr GB, Müller KR (2012) Efficient backprop. In: Neural networks: Tricks of the trade, Springer, pp 9-48

Liao S, Koole G, Van Delft C, Jouini O (2012) Staffing a call center with uncertain non-stationary arrival rate and flexibility. OR spectrum 34(3):691-721

Løkse S, Bianchi FM, Jenssen R (2017) Training echo state networks with regularization through dimensionality reduction. Cogn Comput 9(3):364-378 
Lukoševičius M, Jaeger H (2009) Reservoir computing approaches to recurrent neural network training. Computer Science Review 3(3):127-149

Maass W, Joshi P, Sontag ED (2007) Computational aspects of feedback in neural circuits. PLoS Comput Biol 3(1):e165

Millán-Ruiz D, Pacheco J, Hidalgo JI, Vélez JL (2010) Forecasting in a multiskill call centre. In: International Conference on Artificial Intelligence and Soft Computing, Springer, pp 582-589

Nachar N, et al. (2008) The mann-whitney u: A test for assessing whether two independent samples come from the same distribution. Tutorials in quantitative Methods for Psychology 4(1):13-20

Pascanu R, Mikolov T, Bengio Y (2013) On the difficulty of training recurrent neural networks. In: International conference on machine learning, pp 13101318

Pearson K (1895) Vii. note on regression and inheritance in the case of two parents. P R Soc London 58(347-352):240-242

Shi Z, Han M (2007) Support vector echo-state machine for chaotic time-series prediction. IEEE T Neural Networ 18(2):359-372

Statista Research Department (2021) call center market. https://www. statista. com/statistics/880975/global-contact-center-market-size/, accessed: 2021-06-15

Steckley SG, Henderson SG, Mehrotra V (2005) Performance measures for service systems with a random arrival rate. In: Proceedings of the Winter Simulation Conference, 2005., IEEE, pp 10-pp

Sun Y, Peng Y, Chen Y, Shukla AJ (2003) Application of artificial neural networks in the design of controlled release drug delivery systems. Adv Drug Deliver Rev 55(9):1201-1215

Tandberg D, Easom LJ, Qualls C (1995) Time series forecasts of poison center call volume. J Toxicol Clin Toxic 33(1):11-18

Taylor JW (2008) Exponentially weighted information criteria for selecting among forecasting models. Int J Forecasting 24(3):513-524

Taylor JW (2010) Exponentially weighted methods for forecasting intraday time series with multiple seasonal cycles. Int J Forecasting 26(4):627-646

Wallace RB, Whitt W (2005) A staffing algorithm for call centers with skill-based routing. Manuf Serv Op 7(4):276-294

Weng SS, Liu YH (2006) Mining time series data for segmentation by using ant colony optimization. Eur J Oper Res 173(3):921-937

Zar JH (2005) Spearman rank correlation. Encyclopedia of biostatistics 7

Zou H, Hastie T (2005) Regularization and variable selection via the elastic net. J Roy Stat Soc B 67(2):301-320 